\title{
CALDER EN BARCELONA Y MADRID: JOAN MIRÓ COMO MEDIADOR ARTÍSTICO*
}

\author{
Muriel GÓMEZ-PradAs ${ }^{1}$ \\ Universitat Oberta de Catalunya
}

\begin{abstract}
El objetivo de este artículo es mostrar cómo, gracias a las conexiones individuales y sociales, en la España de la década de 1930 se organizaron exposiciones de artistas de la vanguardia internacional. Tomaremos como caso de estudio el de Alexander Calder que, con la mediación de Joan Miró (re)presentó su performance Cirque Calder en Barcelona (organizado por ADLAN, 1932, 1933) y Madrid (Residencia de Estudiantes, 1933). Una visita propiciada por Miró y que contó con Sebastià Gasch y Ramón Gómez de la Serna no sólo como críticos de la recepción de su obra, sino como verdaderos avaladores de su circo como propuesta artística. Artistas internacionales inscritos en la narrativa del arte moderno de nuestro país a partir de estrechos vínculos personales.
\end{abstract}

Palabras clave: ADLAN; Miró; Calder; vanguardia; circo; escultura; Gasch; exposiciones.

CALDER IN BARCELONA AND MADRID: JOAN MIRÓ AS ARTISTIC MEDIATOR

The aim of this article is to show how, thanks to individual and social connections, exhibitions of artists of the international avant-garde were organized in Spain during the 1930s. As a case study, we will take Alexander Calder, who, with the mediation of Joan Miró (re)presented his Cirque Calder performance in Barcelona (organized by ADLAN, 1932, 1933) and Madrid (Residencia de Estudiantes, 1933). Calder's visit happened under the aegis of Miró, and Sebastià Gasch and Ramón Gómez de la Serna acted not only as critics of the reception of his work, but they also granted credence to his circus as an artistic endeavor. This article addresses the significance of international artists to the narrative of modern Spanish art through close personal links.

Key words: ADLAN; Miró; Calder; avant-garde; circus; sculpture; Gasch; exhibitions.

Cómo citar este artículo / Citation: Gómez-Pradas, Muriel (2019): “Calder en Barcelona y Madrid: Joan Miró como mediador artístico". En: Archivo español de Arte, vol. 92, núm. 368, Madrid, pp. 397-410. https://doi.org/10.3989/ aearte.2019.25.

\section{Introducción}

En este artículo nos centraremos en la vinculación de Alexander Calder (1898-1976) con las ciudades de Barcelona y Madrid en un periodo muy concreto de tiempo en la Segunda República: los años 1932 y 1933. Años que, en el caso de Barcelona, coinciden con el momento en que ADLAN (Amics De L'Art Nou [Amigos del Arte Nuevo])2, agrupación artística y cultural de

\footnotetext{
* Este artículo recoge parte de los resultados de la investigación "ADLAN y la promoción del arte moderno: conexiones nacionales e internacionales" financiada gracias al programa "José Castillejo" para estancias de movilidad en el extranjero del Ministerio de Educación, Cultura y Deporte (referencia CA17/00040).

1 mgomezpr@uoc.edu / ORCID iD: http://orcid.org/0000-0002-1194-1282.

2 Para información detallada sobre ADLAN (1932-1936) véase el monográfico de la revista AC, Cuadernos de arquitectura: ADLAN, testimonio de una época. Barcelona, 1970, $\mathrm{n}^{\circ} 79$ así como las publicaciones: Corredor-Matheos,
} 
vanguardia surgida en la Barcelona de 1932, promovió una serie de actividades artísticas de vanguardia. Y lo haremos focalizándonos en el análisis de material de archivo: especialmente el material efímero que rodea las exposiciones (invitaciones, notas de prensa, carteles, etc.) así como correspondencia y artículos en diarios y revistas de la época, que nos permitirá reconstruir la práctica y recepción de Le Cirque.

Uno de sus principales objetivos de ADLAN, tal como consta en sus estatutos, era promover una serie de manifestaciones culturales encaminadas a la difusión de nuevos lenguajes artísticos: "ADLAN tiene como objetivo la protección y desarrollo del arte, en cualquiera de sus manifestaciones" (Estatutos del grupo Amics De L'Art Nou A.D.L.A.N, 1932, artículo $1^{\circ}$ ). ADLAN contribuyó a dar a conocer entre sus socios una serie de manifestaciones — circo, danza, poesía, exposiciones de objetos populares y cotidianos, etc. - que hasta el momento no tenían ni la categoría ni la consideración de producción artística. Así por ejemplo, una de las actividades, organizada por el crítico Sebastià Gasch (1897-1980) para los socios, fue una excursión en autocar a Mataró el 12 de marzo de 1933 para ver el circo Frediani, una compañía italiana de acróbatas ecuestres ${ }^{4}$. El circo es un tema recurrente que había sido polo de atracción entre numerosos artistas ya desde mediados del siglo XIX, y fue uno de los grandes temas de la modernidad europea. Y ADLAN adoptó el circo como un lenguaje artístico más de la cultura popular, promoviendo actividades en torno al circo como dicha excursión o una exposición colectiva con el circo como temática ${ }^{5}$. Sebastià Gasch fue uno de sus máximos impulsores, tanto como crítico — desde las páginas de revistas y diarios - como protagonista activo y dinamizador de muchas de las iniciativas culturales del momento, muy especialmente de aquellas relacionadas con el mundo del circo. A él se debe, por ejemplo, el intento de crear, adscrito a ADLAN, la asociación "Amics del Circ [Amigos del Circo]"6.

Otra figura unida indisociablemente a la presencia del joven Calder en nuestro país es Ramón Gómez de la Serna (1888-1963). Escritor, periodista y promotor de la vanguardia en Madrid gracias a las tertulias del Café Pombo, era de una generación anterior a Gasch y Calder. Fascinado por el arte del circo, en 1917 escribe la monografía $\mathrm{El} \mathrm{Circo}{ }^{7}$, traducida al francés en 1927, un ejemplar de la cual obraba en poder de Calder ${ }^{8}$.

Existía por tanto en nuestro país el ambiente propicio para la recepción de Calder y Le Cirque o Cirque Calder", que aquí los diarios bautizaron como "El circo más pequeño del mundo" [fig. 1].

\section{Miró, Calder. Barcelona, Madrid}

En los primeros años de la década de 1930 el joven escultor americano Alexander Calder tuvo una intensa presencia en nuestro país, tanto si tenemos en cuenta los viajes de recreo como las performances artísticas relacionadas con su obra Le Cirque o Cirque Calder. Sebastià Gasch, en

\footnotetext{
J. (1988): “ADLAN i el Surrealisme”. En: Surralisme a Catalunya 1924-1936. De l'Amics de les Arts al Logicofobisme. Barcelona: Polígrafa, pp. 49-52; Giralt-Miracle, D. (1992); Minguet, J.M (1997); Guigon, E. (1990): “ADLAN (19321936) et le Surréalisme en Catalogne”. En: Mélanges de la Casa de Velazquez, tome XXXVI/3, pp. 53-80; y Subirana, R. M. (1985): "ADLAN and the artists of Republic. En: Raeburn, M. (ed.) Homage to Barcelona. The City and its Art 1888-1936. London: The Arts Council of Great Britain, pp. 211-225.

${ }^{3}$ Fondo ADLAN, Arxiu Històric del Col·legi Oficial d'Arquitectes de Catalunya (AHCOAC, Barcelona). Todas las traducciones son de la autora.

${ }^{4}$ Para información detallada consultar el Álbum ADLAN, p. 14, Fondo ADLAN, AHCOAC (Barcelona). También véase Gasch, "Una tarda amb la familia Frediani”, El Mirador, no 216, 23 marzo 1933, p. 2; Jané/Minguet, 1998: 22-23 y muy especialmente el catálogo de la exposición $A D L A N$ i el circ frediani (1997).

5 Minguet, 1997: 92.

6 Minguet, 1997: 91, Jané/Minguet, 1998: 22.

7 Existen cuatro ediciones a cargo del autor (1917, 1924, 1943 y 1957) siendo la definitiva, según I. Zlotescu, la de 1943 (Zlotescu, 1998: 813).

8 Según recoge Perl, 2017: 221.

9 Utilizo en este artículo el nombre en francés, en lugar de otras versiones como The Circus, o Calder's Circus, ya que es el nombre con el que primero se le conoció al ser creado y representado en París.
} 


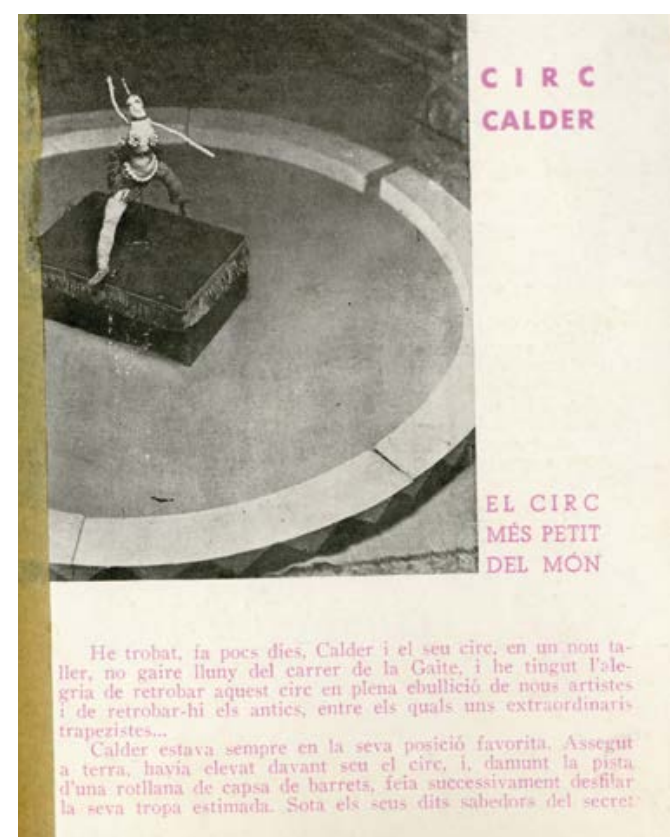

Fig. 1. Folleto exposición Calder. Galería Syra, Barcelona 13 febrero 1933. (C) Arxiu Històric del Col-legi Oficial d'Arquitectes de Catalunya.

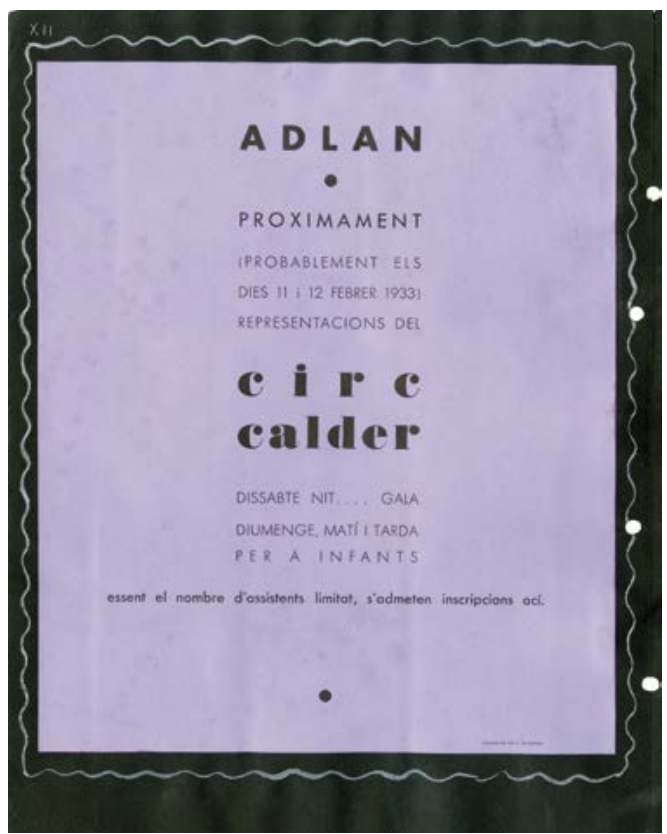

Fig. 2. Poster representación del Circo de Calder. Galería Syra, Barcelona 11 y 12 febrero 1933. (C) Arxiu Històric del Col·legi Oficial d'Arquitectes de Catalunya.

su artículo "L'art d'avantguarda a Barcelona", publicado en el número especial de invierno de 1934 de la revista $D$ 'aci d'allà, escribía que "Este grupo [ADLAN] ha organizado también exposiciones de las obras inéditas del escultor Ángel Ferrant, del pintor Artur Carbonell y del escultor americano Calder, obras todas estas, que, sin esta entidad, no habrían sido expuestas" 10 . En realidad, veremos cómo fue la conexión personal, la incipiente relación de amistad entre Joan Miró (1893-1983) y Alexander Calder, la que propició que en dos intensos años - 1932 y 1933 Calder tuviese una activa presencia en nuestro país [fig. 2].

Como indica Elizabeth Hutton Turner en el catálogo Calder, Mirón ${ }^{11}$, la amistad y afinidad creativa entre ambos artistas se puede documentar perfectamente gracias a las obras, postales, cartas, exposiciones, regalos y viajes que intercambiaron durante casi cincuenta años. El primer contacto entre ambos fue epistolar, tal y como el propio artista rememora en su autobiografía ${ }^{12}$, y así el 10 de diciembre de 1928 Alexander Calder escribía una breve nota de saludo a Joan Miró emplazándolo a verse ${ }^{13}$.

Poco después, en marzo de 1930, Calder realizará su primera visita a Barcelona, como una escala más, junto a Málaga, en su viaje de regreso de Nueva York a París en un barco de la compañía García y Díaz. En esta ocasión, aunque lo intentó, no pudo contactar con Miró ya que éste se encontraba en Mont-roig. Su relación se intensifica y, en verano de 1931 sabemos, gracias a las misivas familiares, como Calder dudaba entre ir a Mallorca con unos amigos o bien ir a Reykjavic a ayudar a su padre ${ }^{14}$ a supervisar la instalación de una estatua. $Y$ aunque en un principio parece

${ }^{10}$ Gasch, 1934: s/p.

${ }^{11}$ Hutton Turner, 2004: 28.

12 Calder, 1966: 92.

13 Carta manuscrita de Alexander Calder a Joan Miró, mezclando francés e inglés, fechada el 10 de diciembre de 1928. Conservada en el archivo de la Fundació Pilar i Joan Miró (FPJM, Palma de Mallorca); copia en CF (Nueva York), carpeta "Correspondence Joan Miró 1929-1947".

${ }^{14}$ Alexander Calder (Sandy como se le conocía familiarmente) era hijo del escultor Alexander Stirling Calder y nieto del también escultor Alexander Milne Calder. 
decidido a ir a Islandia ${ }^{15}$ a ayudarlo, finalmente decide ir a Mallorca ${ }^{16}$, y explica a su familia que ya ha escrito a Miró para que le de consejos y sitios que visitar en Mallorca ${ }^{17}$.

El primer contacto de Miró con el Cirque de Calder fue anterior a octubre de 1931, tal y como se deduce de la carta de Calder a Miró fechada en octubre de 1931, donde le dice que ha invitado al galerista Pierre Loeb pensando que Miró ya le había mencionado las representaciones del circo,

Querido Miró: Como puedes ver, voy a hacer el circo los días 29 y 30 de octubre, y quiero que estés presente. En cualquier caso, ven a vernos cuando llegues a París. Estoy trabajando muy duro, y he hecho muchas cosas con movimiento bastante interesantes, creo (también [Jean] Arp y muchos otros) que te interesará. Invité a Pierre [Loeb] a venir al circo creyendo que quizás hablaste con él al respecto. Puedes decirle que venga si quieres. Mis mejores deseos para los 2, Calder $^{18}$.

Se puede calificar al Cirque Calder $^{19}$ como el primer trabajo artístico ambicioso de Calder ${ }^{20}$, aunque mucha de la crítica del momento pensara lo contrario y lo calificara más como divertimento que como obra de arte ${ }^{21}$. El artista empezó a trabajar en él a finales de la década de 1920, construyendo miniaturas en tres dimensiones de todas las figuras que juegan un papel esencial en un circo (trapecistas, animales, payasos, etc.) en alambre, corcho, retales de tela y diversos materiales "encontrados". Una actuación que en un principio duraba unos escasos 15 minutos pero que, con el tiempo, acabó durando más de 2 horas. Una auténtica performance antes de las performances, con Calder como único actor, y con un asistente que se encargaba de la música. Asistente que en un principio fue el artista japonés Foujita y posteriormente Louisa, su mujer ${ }^{22}$ [fig. 3].

Representación llevada a cabo con pequeñas esculturas de alambre que adquirían vida y movimiento gracias al artista. Y el movimiento será un elemento recurrente al hablar de Calder y su obra. Un espectáculo total, que necesitaba de la participación activa de la audiencia y que contó con la "aprobación" de los surrealistas ya que, como apunta Joy Sperling, "Era un pequeño espectáculo no formal, maravilloso e irreverente que hacía que las audiencias regresaran a estados de la mente subconscientes o infantiles" ${ }^{23}$. Además de añadir la novedad y subversión artística de mostrar una escultura - objeto estático hasta el momento - en movimiento.

Y el circo no era en lo único en lo que trabajaba Calder, tal y como hemos visto que él mismo explica a Miró en la postal de octubre de $1931^{24}$ comentando que está trabajando en objetos en movimiento. Una clara referencia a los móviles en los que trabajaba en ese momento. El nombre francés de "mobile", con el cual se les conoce, el propio artista explicaba por carta al galerista

15 Letter from Louise to Darling mum, carta manuscrita fechada el 23 de junio de 1931, CF, carpeta "Letters to family, 1918-1932".

${ }^{16}$ En la CF (Nueva York), carpeta "Letters to family", se conservan dos cartas de Louisa, esposa de Calder, del 10 y 23 de agosto de 1931, escritas desde Paguera (Mallorca) explicando la estancia en Mallorca. Para información detallada del viaje véase su autobiografía, Calder, 1966: 122.

17 Letter from Sandy to Mr. James, carta manuscrita fechada el 28 de julio de 1931, CF (Nueva York), carpeta "Letters to family".

18 Carta de Calder a Miró, carta manuscrita, en francés, fechada en octubre de 1931, FPJM (Palma de Mallorca).

19 Entre la numerosa bibliografía existente destacar Jed Perl (2017), en concreto el capítulo "Cirque Calder"; Cleve Gray (1964); Joy Sperling (1988); y los catálogos Alexander Calder. The París years 1926-1933. Whitney Museum, Centre Pompidou, and Yale University Press, 2008 y Alexander Calder, circus drawings, wire sculpture and toys. Museum of Fine Arts, Houston, Texas 1964. Actualmente, el circo de Calder forma parte de la colección del Whitney Museum, Nueva York (numero registro 83.36.1-72).

20 Joy Sperling, 1988: 16.

21 "The Art galleries. Spring, Circus and Sport", The New Yorker, c. 1936 en relación a la instalación del Circo en la Galeria Pierre Matisse en 1936. Se conserva un ejemplar en los archivos de la Calder Foundation (CF, Nueva York), carpeta "Calder Circus".

${ }^{22}$ Louisa James (1905-1996). En los archivos de la Calder Foundation se conserva un documento realizado por Calder en 1949 para su mujer Louisa y sus hijas Sandra y Mary con instrucciones, notas y dibujos sobre cómo llevar a cabo una actuación del circo. CF (Nueva York), carpeta "The Circus".

23 Joy Sperling, 1988: 22.

24 Véase nota 18. 
Fig. 3. Fotografía del Calder y su mujer en el álbum de retratos de conferenciantes de la Residencia de Estudiantes. (C) Biblioteca Nacional de España.

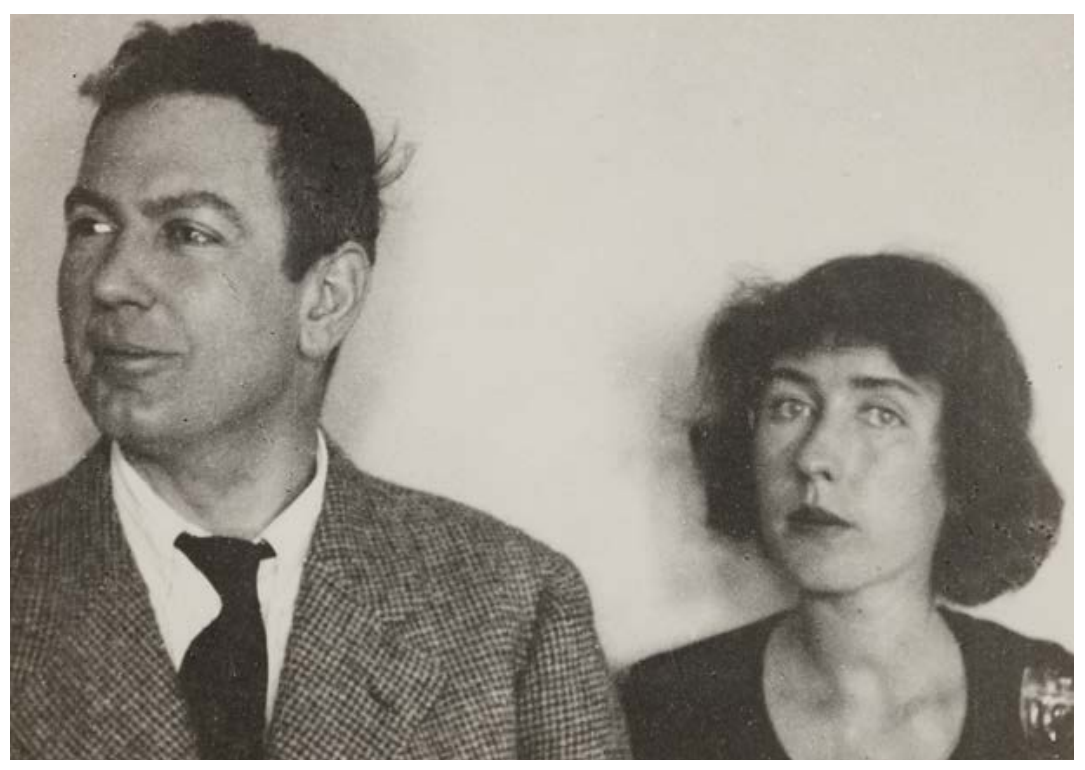

Julien Levy ${ }^{25}$ que se lo había "proporcionado" Marcel Duchamp. Estos "mobile”, que podían llevar motor o no, se caracterizaban por introducir el movimiento de manera explícita y real, obras que jugaban con el movimiento y el equilibrio de volúmenes en suspensión, objetos que podemos calificar de pura abstracción. Los mobiles se exhibieron por primera vez en la exposición individual Calder: ses mobiles en la Galeria Vignon de París del 12 al 19 de febrero de 1932.

La primera performance del Cirque en nuestro país será en septiembre de 1932, durante la estancia de catorce días de Calder en Barcelona ${ }^{26}$. Aunque en realidad la primera actuación no tuvo lugar en Barcelona sino en uno de los lugares más importantes para Miró: su masía en Montroig, a donde Calder llegó el 12 de septiembre de 1932: “[...] el circo emocionó profundamente. Como emocionó al niño mayor que es el pintor Miró y a los cincuenta campesinos de Mont-roig ante los que Calder dio recientemente una representación" 27.

La función del Cirque Calder en Mont-roig fue para Miró, sus amigos, así como sus vecinos y payeses. El propio Miró hace referencia a esta actividad artística en Mont-roig en una carta dirigida a Sebastià Gasch $^{28}$ en la que le comenta que los Calder están encantados de los días que han pasado con él y Prats en Barcelona y le sugiere que vaya a Mont-roig, para ver la performance del circo y así poder escribir un artículo interesante. No queda claro si finalmente Gasch asistió o no a la representación de Mont-roig ${ }^{29}$, pero sí veremos que fue su máximo cronista, escribiendo sobre el circo de Calder no solo un artículo, sino diversos en medios como Mirador, AC y La Publicitat, sobre la (re)presentación de Calder y su circo tanto en Mont-roig como en Barcelona.

A su regreso a Barcelona en septiembre de 1932, Calder vuelve a realizar una representación, sólo para socios, en el hall del GATCPAC. Todos los "adlan" asistieron a estos eventos de Barcelona: Prats, Ferrant, Sindreu, Gasch, etc. y Miró probablemente ${ }^{30}$. Además, Calder exhibe uno de sus móviles en la vitrina del hall del GATCPAC. No se sabe con certeza qué móvil fue expuesto

${ }^{25}$ Carta de Calder a Levy, marzo de 1932, manuscrita. Julien Levy Records, Philadelphia Musseum of Art (JLP, Filadelfia) archivador 8 carpeta 4. Sobre el significado y uso de la palabra "mobile" véase Perl, 2017: 407, donde encontramos una descripción más larga y detallada que la que el propio artista proporciona a Levy.

${ }^{26}$ Calder, 1966: 138.

27 Gasch, 1932b: 5.

${ }_{28}$ Carta de Miró a Sebastià Gasch, Mont-roig 12 septiembre 1932. Recogida en Minguet/Montaner/Santanach, 2009: 489.

${ }^{29}$ Minguet/Montaner/Santanach, 2009: 489.

${ }^{30}$ La Publicitat, 24-IX-1932: 3. 


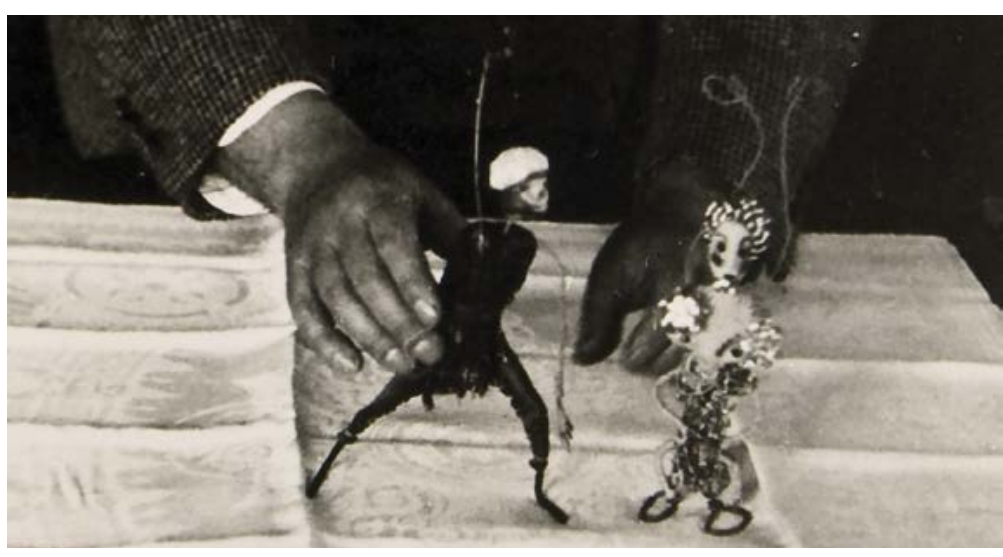

Fig. 4. Imagen de la representación de "El circo más pequeño del mundo". Residencia de Estudiantes, Madrid 1 y 2 de febrero 1933.

(C) Biblioteca Nacional de España.

en Barcelona. Podría ser uno de los dos que ilustraban el artículo de Gasch en la revista $A C$ : Object with red disc [Objeto con disco rojo] (1931) ${ }^{31}$ o Object with red ball [Objeto con bola rojo] $(1931)^{32}$. Pero no coinciden con el que ilustra el artículo del mismo autor para Mirador, titulado Mobile (1932) y actualmente desaparecido ${ }^{33}$. También podría ser uno de los dos móviles, también de 1931, que se conservan en el MACBA ${ }^{34}$ y que pertenecieron al arquitecto del GATCPAC Germán Rodríguez Arias, quien parece ser que conoció a Calder en Barcelona precisamente en $1932^{35}$. Todos ellos caracterizados por el uso de formas geométricas puras de colores primarios, pura abstracción en movimiento. Al no conservarse fotografías ni documentación de la muestra, no podemos concluir si el móvil expuesto en el hall del GATCPAC era uno de estos cinco o no .

$\mathrm{Al}$ año siguiente, el matrimonio Calder regresará a Barcelona, añadiendo en su itinerario a la ciudad de Madrid gracias al buen hacer de Miró. Según explica el propio Calder ${ }^{36}$, fue gracias a la mediación de Joan Miró con Ramón Gómez de la Serna ${ }^{37}$ que Calder recibió la invitación para actuar con su circo para la Sociedad de Cursos y Conferencias en la Residencia de Estudiantes en Madrid ${ }^{38}$ [fig. 4]. Hecho que se confirma gracias a una carta de Miró a Prats fechada el 4 de septiembre donde el propio artista explica que "he escrito también a Gómez de la Serna sugiriéndole esta idea" ${ }^{39}$. Unos días después, concretamente el 9 de septiembre, Miró le confirma por carta a Prats que "Acabo de recibir unas palabras de Ramón Gómez de la Serna diciendo que le interesa mucho la idea que yo le propongo para el circo [...]. Se podría hacer de manera que la exhibición de Barcelona coincidiese con la de Madrid, así iría mejor para el viaje y transportes"40. Se corrobora así el destacado papel de Miró como mediador, como enlace y catalizador cultural ${ }^{41}$,

31 Actualmente se conserva la colección del Whitney Museum of American Art, número de registro 86.49a-c.

32 Colección privada.

33 Hutton Turner, 2004: 282 y nota 13.

34 MACBA, $n^{\circ}$ de registro 0020 y 0021.

35 Véase < https://www.macba.cat/ca/sense-titol-0020> [5 de junio de 2018]. Se ha podido comprobar que en 1933 dicho arquitecto fue uno de los socios del GATCPAC que recibió invitación personalizada para asistir a la exposición del artista en 1933 (Álbum ADLAN, p. 12, Fondo ADLAN, AHCOAC, Barcelona). Y precisamente en 1933 uno de ellos ( $\mathrm{n}^{\circ}$ MACBA 0020) ya obraba en su poder, siendo expuesto en el stand del GATCPAC de la Feria de Muestras de Barcelona (junio de 1933) (fotografía C27-170-6-6, archivo GATCPAC, AHCOAC, Barcelona).

${ }_{36}$ Calder unpublished manuscripts, 18 de junio de 1956, p. 147, CF. Citado en Hutton Turner, 2004: nota 16.

37 Ambos se conocían desde hacía años, del viaje que Miró hizo a Madrid en 1928 y en el cual se relacionó con el grupo de La Gaceta Literaria al cual estaba vinculado el escritor. Gómez de la Serna, en el capítulo dedicado al Suprerrealismo de su destacado libro Ismos (1931), elogia la obra de Miró.

${ }^{38}$ Se conserva una relación de los gastos de su estancia en el Archivo General de la Administración (AGA) de Alcalá de Henares, caja 9180, signatura 31/109180.

39 Carta de Miró a Prats, Mont-roig, 4 de septiembre de 1932. Minguet/Montaner/Santanach, 2009: 487.

40 Carta de Miró a Prats, Mont-roig, 9 de septiembre de 1932. Minguet/Montaner/Santanach, 2009: 488.

${ }^{41}$ Cómo introducción al concepto de mediador cultural aplicado a procesos artísticos véase el artículo de Meylaters, et alt, 2017, pp. 67-91. 
conectando intereses (circo como actividad artística) y personalidades (Calder y Gómez de la Serna). Un papel que también nos habla, y mucho, de la propia predisposición de Miró hacia este tipo de obras.

Con Gómez de la Serna como orador, Calder recreará su Cirque en la Residencia de estudiantes durante dos días, concretamente los días 1 y 2 de febrero de 1933 para los miembros de la Sociedad: "Como el espectáculo no puede ser dominado de una vez más que por ochenta o cien personas, Calder repetirá su función varias veces, para que todos los socios de los Cursos y Conferencias puedan asomarse al pequeño circo"42. La Sociedad de Cursos y Conferencias ${ }^{43}$ fue creada en 1924, una sociedad dependiente de la Residencia de estudiantes que organizaba actos culturales, tanto de científicos como de artistas nacionales e internacionales. Un foro abierto a las vanguardias, una ventana cultural en la capital de lo que ocurría en Europa.

Calder conocía y admiraba el libro El circo (1917) de Gómez de la Serna, del cual ya hemos comentado que tenía un ejemplar de la edición francesa de 1927, y esta breve estancia en Madrid le permitió asistir a una de las conocidas tertulias organizadas por Gómez de la Serna en el café Pombo de Madrid ${ }^{44}$, así como le dio la oportunidad de visitar su estudio ${ }^{45}$. De su paso por Madrid se conserva un dibujo que realizó para Natalia Jiménez Cossio ${ }^{46}$, hija de Alberto Jiménez Fraud, el director de la Residencia de Estudiantes en aquel momento.

Además, por las fotografías conservadas ${ }^{47}$, parece ser que Calder también aprovecho para exponer alguno de sus móviles en la Residencia de Estudiantes [fig. 5], tal y como ya había hecho en Barcelona el año anterior. De este evento se conserva una fotografía que nos permite identificar el móvil como Mobile (1933) ${ }^{48}$. Un móvil característico de la década de 1930: construcciones con formas geométricas que se mueven rítmicamente, creando formas especulativas y variables en el espacio.

Tras Madrid el artista vuelve a Barcelona, donde además de volver a realizar la performance del Cirque Calder ${ }^{49}$ exhibirá sus dibujos y esculturas en una exposición organizada por ADLAN en la Galería Syra en febrero de 1933 gracias, otra vez, a la mediación de Joan Mirós ${ }^{50}$. Se afianza en este caso Miró como figura poliédrica, como vínculo, como mediador cultural, como figura que facilitó y contó con diversas complicidades para dar a conocer en nuestro país a un joven representante de la vanguardia internacional afincada en París como Calder.

En relación a la organización de esta exposición, debemos constatar que en un principio las fechas previstas eran enero de $1933^{51}$, aunque dependían de las fechas en que Gómez de la Serna organizara el acto en Madrid, tal como hemos visto que explica Miró y como se deprende de la carta de la carta que Calder dirigió a Joan Prats fechada en diciembre de $1932^{52}$. Entre la co-

${ }^{42}$ Folleto "El circo más pequeño del mundo", los días 1 y 2 de febrero de 1933, a las seis y media de la tarde en la residencia de estudiantes, Pinar 21. CF (Nueva York), carpeta "Residencia de Estudiantes, Madrid 1933".

43 Para información sobre la historia y las actividades organizadas por esta entidad vinculada a la Residencia de Estudiantes véase Ribagorda, Á. (2009): "El Comité Hispano-inglés y la Sociedad de Cursos y Conferencias de la Residencia de Estudiantes (1923-1936)". En: Cuadernos de Historia Contemporánea, 30, pp. 273-291, doi:10.5209/CHCO.7580

44 Donde firma como visitante, según recoge Martínez-Collaso, 1997: 61.

45 La visita al estudio "ramoniano", se cita en el catálogo Los ismos de Ramón Gómez de la Serna y un apéndice circense, Diaz Sánchez/Bonet/et alt, 2002: 451.

${ }^{46}$ Este dibujo, un autorretrato de Calder y su mujer con una de las características maletas en las que transportaba su circo, fue publicado en la revista Poesía, no 17-18 publicada en 1978, así como en el libro Álbum de Natalia II, de la propia Natalia Jiménez Cossio. He de agradecer a Alfredo Valverde, del Centro de Documentación de la Residencia de Estudiantes dicha información.

47 "Retratos de conferenciantes en la Residencia de Estudiantes", Signatura 17/87 Sede Recoletos, Biblioteca nacional de España.

48 Actualmente en el Musée National Fernand Leger (Biot, Francia), tal y como aparece referenciado en el catálogo El universo de Calder (IVAM, 10 septiembre - 15 noviembre 1992), p. 270.

49 En el Álbum ADLAN, Fondo ADLAN, AHCOAC (Barcelona) se conservan invitaciones tanto de las representaciones del circo como de la exposición.

${ }^{50}$ Letter Louisa to Mother, manuscrita, diciembre de 1932, CF (Nueva York), carpeta "Letters to family".

51 Según consta en el documento con la programación de ADLAN (de 1932 a 1935) conservada en el Fondo ADLAN, AHCOAC (Barcelona). Documento mecanografiado con añadidos a lápiz.

52 Carta de Calder a Joan Prats, manuscrita, diciembre de 1932. Fondo ADLAN, AHCOAC (Barcelona). 


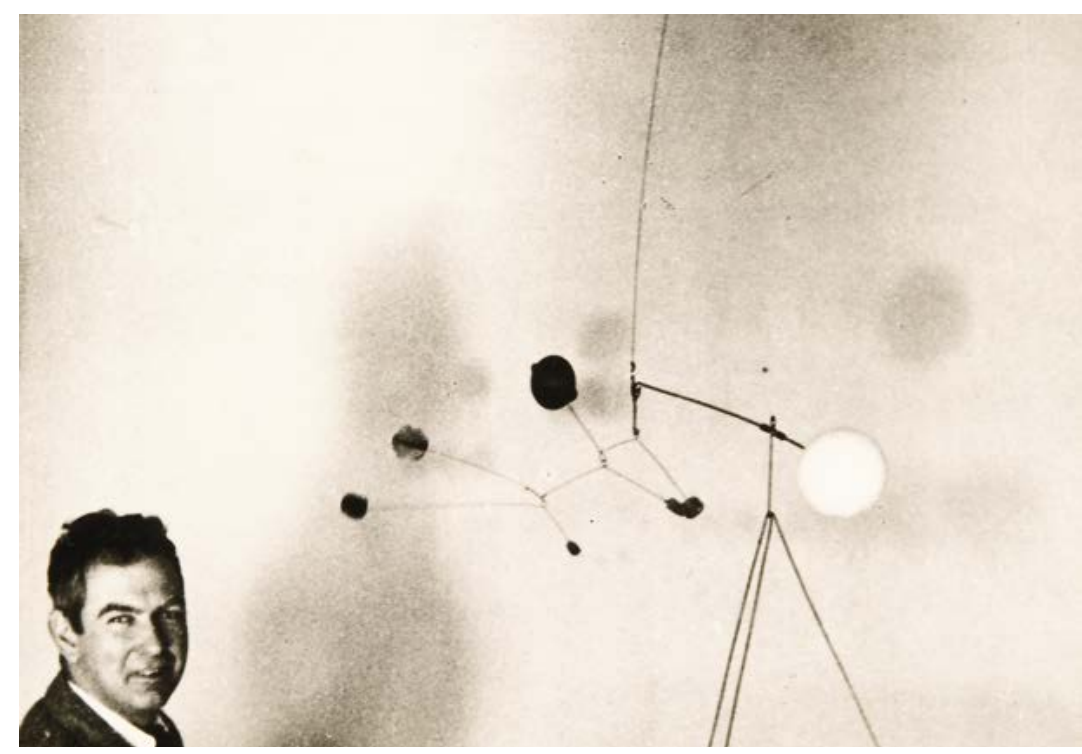

Fig. 5. Alexander Calder en la Residencia de Estudiantes, Madrid 1 y 2 de febrero 1933.

(C) Biblioteca Nacional de España.

rrespondencia de Miró a Prats ${ }^{53}$, vemos como en diciembre ya hay una aproximación real a las fechas - la primera semana de febrero en Madrid y la segunda en Barcelona—, aunque no será hasta poco tiempo antes cuando, en una carta de Calder a Prats escrita ya desde Madrid ${ }^{54}$, éste le confirma que el 11 y el 12 de febrero le parecen fechas correctas. Gracias a este intercambio de misivas hemos podido señalar como la idea de exhibir los dibujos junto a los móviles parte del propio Calder, siguiendo una idea previa de Miró: "Miró me dijo que una noche había mostrado sus cuadros en casa ${ }^{55}$. Si lo desea, puedo traer algunos dibujos que hice recientemente y mostrarlos de la misma manera" ${ }^{\text {", }}$, idea a la cual Prats responde de manera afirmativa y entusias$\operatorname{ta}^{57}$. Además de la exposición en la Galería Syra, también se llevaron a cabo dos actuaciones del circo los mismo días ${ }^{58}$. Gasch, en su artículo "Un ianqui a Barcelona" hacer referencia a algunos de los "adlan" que asistieron, como Carles Sindreu y Mirós9 [fig. 6].

No se conserva ninguna relación de los dibujos y móviles que Calder expuso en la Galería Syra, aunque por las referencias aparecidas en la prensa, como por ejemplo el artículo de Gasch "Un ianqui a Barcelona" publicado en Mirador, podemos hacernos una idea: no sólo se expusieron los de temática circense - como "Entrenador de caballos con aro" $(1931)^{60}$ que ilustra el articulo-, sino también algunos de sus dibujos abstractos. Aunque, tal y como explica el crítico de arte parece ser que no tuvieron un gran éxito (como mínimo de crítica): "Objetos móviles y circo. Este es el verdadero Calder. Mucho más auténtico que el de los dibujos, demasiado influenciados por Miró y Picasso. Excepción hecha de los deliciosos dibujos de circo [...]"61.

${ }^{53}$ Carta de Miró a Prats, París, 9 de diciembre de 1932. Minguet/Montaner/Santanach, 2009: 490.

${ }^{54}$ Carta de Calder a Joan Prats, sin fechar, manuscrita en papel con el membrete del Hotel Majestic de Madrid. Fondo ADLAN, AHCOAC (Barcelona).

55 El 27 de noviembre de 1931, Joan Miró realizó una exhibición privada de sus obras más recientes en su casa del Passatge del Credit.

56 Carta de Calder a Joan Prats, manuscrita, diciembre de 1932. Fondo ADLAN, AHCOAC (Barcelona).

57 Borrador de Prats a Calder, manuscrita, sin fechar, Fondo ADLAN, AHCOAC (Barcelona).

58 En el Álbum ADLAN, Fondo ADLAN, AHCOAC (Barcelona) se conservan invitaciones de los dos días, así como información sobre el precio del acto. En un principio estaban previstas tres actuaciones: la noche del 11, y por la mañana y la tarde del día 12, aunque finalmente la sesión de la mañana se anuló.

59 Gasch, 1933b: 7.

${ }^{60}$ Actualmente en la CF (Nueva York).

${ }^{61}$ Gasch, 1933c: 7. 
Fig. 6. Invitación inauguración exposición Calder.

Galerías Syra, Barcelona 13 febrero 1933. (C) Arxiu Històric del Col·legi Oficial d'Arquitectes de Catalunya.

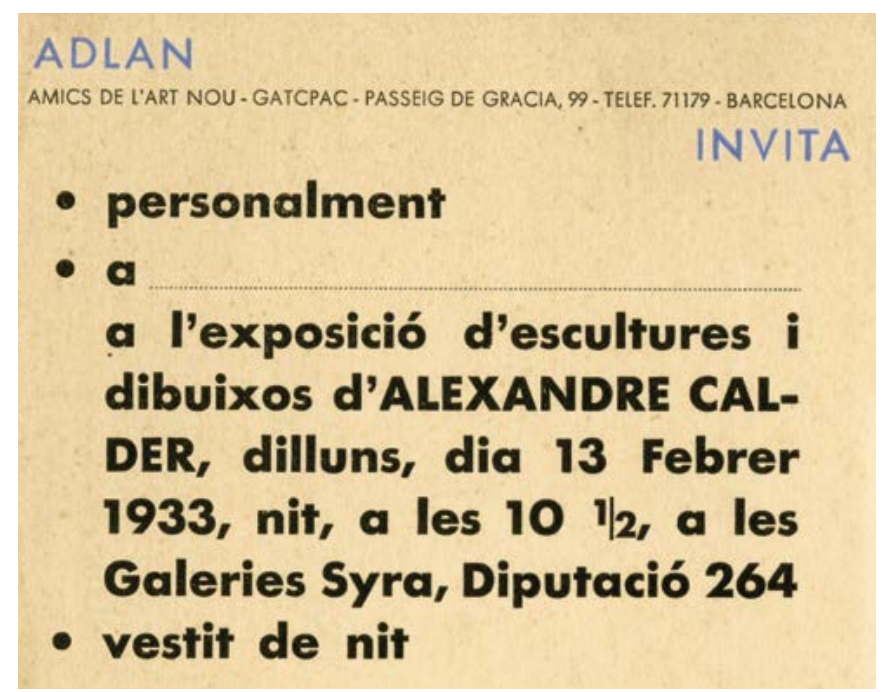

Pero Calder sí que triunfó con los “objetos". Es interesante observar como Gasch habla de estos objetos móviles en diversas de sus crónicas del Mirador, gracias a las cuales podemos reconstruir el tipo de objetos que se exhibieron: móviles sin ningún tipo de mecanismo mecánico, únicamente movidos por la propia inercia de la gravedad, por el azar, por el aire o por la propia intervención humana, que en la galería aparecían dispuestos bien fuera colgados bien en pedestales ${ }^{62}$. En su crónica, Gasch inclu ye una valoración de esta obra, diferenciando los objetos móviles de Calder de los objetos encontrados de Ferrant o Miró, gracias al añadido de dos nuevas variantes: el movimiento y el hecho de no ser imitativos sino pura abstracción, aspectos que los hace únicos,

Los ADLAN ya han visto objetos. Joan Miró y Ángel Ferrant los han mostrado otras veces. Los de Calder, al contrario, son objetos que se mueve, combinaciones de alambres y pesos bien equilibrados que blanden largo rato bajo un leve impulso; poleas y excéntricas que hacen y deshacen figuras geométricas con variedad de formas; objetos deliciosamente inútiles y curiosamente decorativos que no recuerdan ni quieren recordar nada: puras abstracciones ${ }^{63}$.

\section{Calder en Barcelona y Madrid. Recepción crítica}

A pesar de ser unas acciones minoritarias, y en 1932 exclusiva para socios, las intervenciones de Calder en Barcelona tuvieron cierta repercusión en la prensa, como todas las actividades avaladas por los ADLAN. Así como también la tuvo en Madrid, al ser una actuación respaldada por la figura de Gómez de la Serna.

El máximo defensor, tanto de las representaciones del circo como de las exposiciones fue, como ya hemos ido constatando, el crítico catalán Sebastià $\mathrm{Gasch}^{64}$, tanto desde las páginas $\mathrm{La}$ Publicitat como desde Mirador y AC. El primer artículo que Gasch escribía sobre la presentación de "El circ més petit del mon [El circo más pequeño del mundo]" en septiembre de 1932 apare-

62 Gasch, 1933c: 7.

${ }^{63}$ Gasch, 1933a: 7.

64 Para información detallada sobre uno de los críticos de arte más destacados de la vanguardia barcelonesa véase Gasch, Sebastià (1987): Escrits d'art i d'avantguarda. Barcelona: Edicions Moll, y Minguet, J.M. (1997): Sebastià Gasch. Crític d'art i de les arts de l'espectacle. Barcelona: Departament de Cultura, Generalitat de Catalunya. 
ce publicado en las páginas de un diario de larga tradición catalanista e intelectual, La Publicitat $t^{65}$, en un artículo en el que destaca que

Este escultor americano ha aislado del circo sus elementos más característicos, los elementos estándar que podríamos llamar tópicos: el hombre más fuerte del mundo, el ecuyere, el domador, el hombre que se traga sables, el equilibrista, los trapecios volantes, el crottin, los ruidos, los silencios: la música que enmudece durante los ejercicios peligrosos, etc. Y con todo esto ha hecho una síntesis definitiva del circo. El arquetipo. La quintaesencia. El purismo del circo, como decía el escultor Ferrant. Todos los tópicos del circo en el estado puro, con su calidad standard singularmente acentuada ${ }^{66}$.

También es de Gasch la reseña sobre la obra de Calder que publica la revista $A C$, dependiente del GATPAC y que según apunta Giralt-Miracle 67 "ADLAN aprovechaba como tribuna". Con el artículo "El escultor americano Calder", Gasch glosa la obra del artista y lo inscribe dentro de la corriente Abstraction Création junto a artistas como Arp, Helion y Mondrian, tal y como aparecía en la nota informativa que desde ADLAN se enviaba a la prensa ${ }^{68}$. En este artículo destacaba que "Las obras de Calder son bellas en sí, no por comparación, y tienen una belleza autónoma. Son pura creación, como creación es una flor o una fruta. Puras invenciones de formas. Creación, no imitación"69.

Y en Mirador Gasch escribe en 1932 un extenso artículo ilustrado en que hace una clara defensa de la obra de Calder como escultor y de las figuras utilizadas en sus performances de Le Cirque también como elementos escultóricos: "Los muñecos del circo de Calder son un prodigio de forma y color. Son una pura delicia escultórica. Y su mecanismo es hijo de un ingenio formidable. Pero por encima de todo, hay que señalar la frescura y la infinita poesía de este espectáculo que nos hace retroceder a los buenos años de la infancia" 70 .

El regreso de Calder a Barcelona en febrero de 1933 sí tuvo más repercusión en la prensa del momento. Y, aunque La Vanguardia sólo le dedico tres líneas en su sección "Música y teatros"71 igual que El día gráfico ${ }^{72}$, encontramos otras referencias mucho más detalladas y documentadas. Así por ejemplo, La Veu de Catalunya ${ }^{73}$, uno de los diarios catalanes más importantes de la época, publica una noticia sobre la representación del circo en febrero de 1933 en la que se hace hincapié en el hecho de que esta vez las actuaciones del "Circ més petit del món" serán públicas, debido al éxito que tuvieron unos meses antes los pases privados que el artista realizó de manera exclusiva para los socios de ADLAN. Y recomienda a todos los amantes del circo que no se lo pierdan, ya que "Calder, poeta y humorista, nos ofrece con su circo una síntesis caricatural y lírica, con una fantasía inigualada de alto valor plástico" "74. O el artículo anónimo de Mirador fechado el 9 de febrero de 1933, en el cual también se incluye un anuncio ${ }^{75}$ sobre las representaciones del circo [fig. 7].

La Publicitat trata diversas veces el tema, como en el artículo del 9 de febrero de $1933^{76}$ o la breve nota explicativa del 12 de febrero de $1933^{77}$ sobre el éxito de la representación la noche

${ }^{65}$ Aunque el artículo va sin firmar, sabemos que era de Gasch ya que se trata de un extracto de uno más extenso que publico en el número 191 de la revista Mirador.

66 Gasch, 1932b: 5.

67 Giralt-Miracle, 1992: 99.

${ }^{68}$ Se ha de resaltar que la información relativa al grupo Abstraction Création aparece en la nota informativa que se enviaba a la prensa. Véase Álbum ADLAN, p. 10, Fondo ADLAN (AHCOAC, Barcelona).

${ }^{69}$ Gasch, 1932a: 43.

70 Gasch, 1932b: 5.

${ }^{71}$ La Vanguardia, 11 de febrero de 1933, p. 25.

72 "Presentación del Circo en miniatura de Alejandro Calder", El día gráfico, 12 de febrero 1933, s/p.

73 Sobre la importancia de este diario, publicado en catalán, en la vida cultural y política del momento véase Figueres, J. M. (2014). La Veu de Catalunya (1899-1937). Barcelona: Base edicions.

74 "Calder y el circ més petit del mon". En La Veu de Catalunya, febrero de 1933, p. 5.

75 De la inserción de este anuncio se conserva la factura de 12,15 pesetas. Fondo ADLAN, AHCOAC (Barcelona).

76 "El "circ més petit del món", de l'escultor Alexander Calder, a Barcelona". Mirador, 9 de febrero 1933.

77 La Publicitat, 12 de febrero de 1933, p. 6. 
Fig. 7. Artículos del Mirador y $L a$ Publicitat, 9 de febrero de 1933. Resumen de prensa realizado por la secretaria de ADLAN Adelita Lobo. (C)Arxiu Històric del Col·legi Oficial d'Arquitectes de Catalunya.

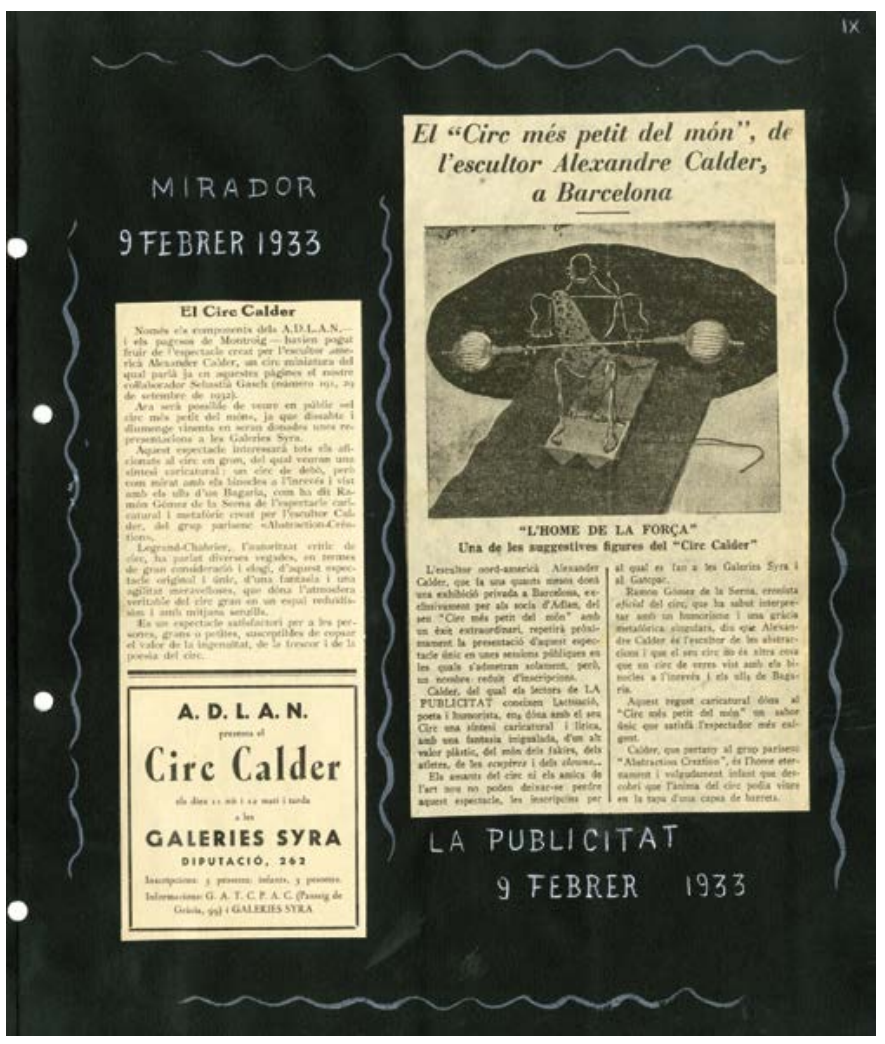

anterior de "El circ més petit del món [el circo más pequeño del mundo]" en las Galerías Syra y organizado por ADLAN. Unos días más tarde, el mismo medio publica una breve y poética nota de Focius ${ }^{78}$ o el artículo "Alexander Calder, escultor"79, firmado por Gasch, donde repite ideas ya exploradas en sus anteriores escritos: purismo del circo, diversión, lirismo, obra plástica, dinamismo y otros lugares comunes. Pero será en Mirador donde encontramos las crónicas más extensas y fundamentadas de Gasch, en las cuales por ejemplo diferenciará los móviles de Calder en relación a la poética de los ready-made utilizada por Ferrant y Miró ${ }^{80}$ como ya hemos visto, con verdaderas aportaciones críticas a la obra de Calder — tanto dibujos como objetos y circorelacionando los dibujos con Miró y Picasso ${ }^{81}$.

También en el seminario satírico barcelonés $E l$ Be Negre $e^{82}$ encontraremos referencias a Calder. El Be Negre ${ }^{83}$ nació con la proclamación de la Segunda República y, entre 1931 y 1936, destacó por sus críticas de tono mordaz, sarcástico y provocador. Fue muy crítico con todo lo que era y significaba ADLAN en el panorama cultural de la ciudad. Y en una breve nota anónima, como la mayoría de las publicaciones del semanario, relata lo que califica como "primera exhibición del grupo ADLAN en GATCPAC" y tirando de ironía aprovecha en realidad para arremeter contra Gasch explicando como "el escultor Calder presentaba su circo en miniatura cuyo recrítico Gasch ya dijo de ella todo lo necesario en las páginas de Mirador"

\footnotetext{
${ }^{78}$ Focius, 1933: s/p. Focius era el pseudónimo que utilizaba J.V. Foix en La Publicitat.

${ }^{79}$ Gasch, 1933b: 9.

80 Gasch, 1933a: 7.

81 Gasch, 1933c: 7.

82 "Els tocats de l'ADLAN". En El Be Negre, any 5, n 197, 3 abril 1935, p. 2.

83 Sobre la importancia de este semanario en la vida cultural y política catalana véase Solà i Dachs, Ll. (1977): El Be Negre i els seus homes. Barcelona: Edhasa.

${ }^{84}$ Be negre, octubre 1932, p. 2.
} 
$\mathrm{Al}$ año siguiente, este mismo seminario persiste en aprovechar cualquier excusa para continuar con las críticas a Gasch, como en la parodia crítica titulada "El plaga de Mr. Calder. Un metre de més o de menys [La plaga de Mr. Calder. Un metro de más o de menos]" publicada el 28 de febrero de 1933. En esta parodia, típica del Be Negre, sobre un hipotético encuentro del periodista con Calder, aprovechan para cargar contra Gasch, poniendo en boca de Calder la siguiente hipótesis sobre la "verdadera" razón del nacimiento del circo,

Sebastià Gasch había terminado las bailarinas y los chansonniers del país y ya no sabía de qué hacer artículos para poder mantener lícitamente el título de recrítico de vuestro music-hall. Fue entonces que los del "ADLAN" me dirigieron una afectuosa carta rogándome que me inventara algo para salir, todos juntos, airosos de este serio compromiso y de ahí el nacimiento de mi circo miniatura ${ }^{85}$.

A expensas de Calder, las bromas continúan meses más tarde en dicho seminario, y así en marzo aun publican que los artistas de adlan han invitado a una "costellada" a Calder ${ }^{86}$ y en abril bromean nominándolo como Miss GATCPAC ${ }^{87}$.

Las crónicas desde Madrid no difieren demasiado de las realizadas en Barcelona. La noticia de Miguel Pérez Ferrero para el Heraldo de Madrid ${ }^{88}$, uno de los diarios más populares y de mayor tiraje en la España de la época, iba ilustrada con una foto del matrimonio Calder entre Ramón Gomez de la Serna y el propio escritor y periodista, se hacen constantes referencias a la presencia física del artista, de cómo manipula a todas las figuras, a cómo mantiene una "constante presencia en la pista", para acabar preguntando al espectador "no se pueden establecer comparaciones. No se puede dilucidar. ¿Qué es mejor, Calder mismo o su circo?" 89 Y desde las páginas de $E l$ Sol $l^{90}$, diario publicado entre 1917 y 1939 y que aportó aires renovados al periodismo de la capital, la poética crónica firmada por A. R. de L. explica las sensaciones que vivió al ver dicho espectáculo,

Lo de menos es la precisión de cálculo en este o el otro ejercicio de riesgo. Lo asombroso, lo inaudito es como el arte y la ingenuidad noble de un espíritu de chiquillo como es el de Calder, se ha valido para reducir en unos simples tapones de corcho, engarzados en un alambre, toda la soberbia vanidad, y la fastuosa fanfarronería, y la misma muerte, alumbrada de lentejuelas, que rebullen en el circo. Y lo que es mejor, infinitamente mejor, con un regusto de caricatura que pone frío en la risa ${ }^{91}$.

El propio Gómez de la Serna ${ }^{92}$ escribe un artículo ilustrado en el periódico republicano $\mathrm{Luz}^{93}$, en el que hace una pormenorizada y épica relación de todas las figuras que aparecen en la representación llevada a cabo por Calder, destacando la "esbeltez artística" de la representación.

Vemos, por tanto, un cierto cambio en la apreciación crítica de la obra de Calder. En 1932 la mayoría se centraron en el circo como espectáculo, no considerándolo una obra artística sino como un divertimento, haciendo hincapié en su lirismo, a excepción hecha de la crítica de Gasch del 21 de septiembre de 1932, donde sí valora las pequeñas figuras del circo como verdaderas esculturas. Mientras que al año siguiente la percepción evoluciona y pasa a ser valorado como artista, capaz de divertir y conmover con el circo y de realizar unas obras, unos "objetos" únicos como son los

85 "El plaga de Mr. Calder. Un metre de més o de menys". Be Negre, 28 de febrero de 1933, p. 3.

86 Be Negre, 7 marzo 1933, p. 4.

87 Be Negre, 18 abril 1933, p. 4.

${ }^{88}$ Para más información sobre el Heraldo de Madrid, líder de la prensa republicana en España, véase $<$ http://hemerotecadigital.bne.es/details.vm?q=id:0000384902\&lang=en> [11 mayo 2018] y el libro de Toll, Gil (2013). Heraldo de Madrid, tinta catalana para la II República española. Sevilla: Editorial Renacimiento.

89 Pérez Ferrero, 1933: 2.

${ }^{90}$ Para más información véase $<$ http://hemerotecadigital.bne.es/details.vm?q=id:0000182002\&lang=en $>$ [11 mayo 2018]

91 "En la residencia de estudiantes". El Sol 2/2/1933, página 8.

92 Gómez de la Serna (1933). "Calder y su circo". Luz, 1 de febero de 1933, pp. 8-9.

93 Para más información véase http://hemerotecadigital.bne.es/details.vm?q=id:0003494082\&lang=en [11 mayo 2018]. 
móviles. En todas las críticas se repiten conceptos como "invención, no imitación", pureza de formas, alto valor plástico, abstracción pura, etc. para definir, describir y valorar los móviles de Calder. Finalizar destacando cómo la visita del joven artista norteamericano fue facilitada por Miró, pero contó con la total complicidad de Gasch y Gómez de la Serna no sólo como críticos de la recepción de su obra, sino cómo verdaderos avaladores de su circo como propuesta artística.

\section{Conclusiones}

La poética de la obra de Calder del momento se ajustaba perfectamente a las preferencias de "los adlan", ya que ADLAN fue proyecto cultural multidisciplinar y ecléctico, donde una de las manifestaciones de la cultura popular que alcanzó mayor relevancia fue el circo. Aunque en realidad, tal y como hemos podido comprobar, no fue propiamente por ADLAN sino gracias a la amistad de Calder con Miró, que Calder tuvo presencia activa en Barcelona. Así se entiende, además, que en esa misma época también realizara performances de Cirque Calder en Madrid (Residencia de Estudiantes) o que la primera performance en España la realizara precisamente en Mont-roig.

Miró/Calder, unos vínculos personales y artísticos que fueron más allá de los años treinta. A ambos les unía el sentido del humor, la ironía que trasladaban a sus obras así como su constante experimentación, su proceso de simplificación y economía de medios, su poética de lo esencial. Y en los dibujos abstractos que Calder expuso en Barcelona en 1933 la crítica del momento precisamente ya hemos visto cómo se hacía eco de que recordaban "demasiado" a las obras de Picasso y Miró ${ }^{4}$.

Y además de los estrechos vínculos personales que Calder estableció con Miró, a raíz de estas visitas, Sebastià Gasch (a quien cómicamente llamaba Gassko) y Joan Prats pasaron a engrosar su lista de amistades, como se desprende de la correspondencia conservada con ambos que perduró a lo largo de los años.

El trabajo de archivo nos ha permitido documentar la trayectoria de Calder en nuestro país, reconstruir y estudiar las exposiciones y representaciones de su obra, así como la recepción que tuvieron sus proyectos artísticos. Poniéndose así de relieve la importancia de los documentos en la valoración y reconstrucción de la práctica y los procesos artísticos.

Una labor de archivo que en este caso ha posibilitado sacar a la luz el rol de Joan Miró como un verdadero mediador cultural, conectando ciudades y artistas, creando y organizando espacios de confluencia entre artistas nacionales e internacionales, entre narrativas expositivas, entre contextos diversos, pero con un interés común: el arte moderno, el nuevo arte. Miró como catalizador, como enlace, como introductor en nuestro país de Calder, un joven representante de la vanguardia internacional. Una visita propiciada por Miró y que contó con Gasch y Gómez de la Serna no sólo como críticos de la recepción de su obra, sino como verdaderos apoyos, promotores y garantes de su circo como propuesta artística. Unas conexiones y redes sociales cuyo conocimiento nos proporciona una manera diferente de ver y entender la historia de la modernidad.

En Madrid podemos colegir que fue una presentación más institucionalizada, auspiciada por la Sociedad de Cursos y Conferencias de la Residencia de Estudiantes, con una larga trayectoria en la organización de actividades de todo tipo con científicos, intelectuales y artistas internacionales (Marie Curie, Le Corbusier, Theo Van Doesburg, Walter Gropius, Igor Stravinsky, Paul Valery, Louis Aragon, Marinetti, entre otros). Mientras en Barcelona contó con el respaldo de ADLAN, asociación de nueva creación y organizada por un grupo de diletantes con un ambicioso proyecto: mostrar en Barcelona propuestas artísticas significativas de la vanguardia internacional, actividades eclécticas, multidisciplinarias, encaminadas a abrir los límites de lo artístico.

A pesar de ello, no debemos olvidar que el ambiente cultural y artístico mayoritario de ambas ciudades era muy distinto y alejado de esos "experimentos" de vanguardia. Así por ejemplo, la performance de Calder, al igual que la mayoría de las actividades culturales organizadas por ADLAN, tuvo una repercusión positiva en la prensa del momento, mayoritariamente en medios

94 Gasch, 1933c: 7. 
y críticos afines —entre los que sobresale claramente la figura de Sebastià Gasch—, pero en realidad se ha de tener muy presente que eran actividades minoritarias y dirigidas exclusivamente a socios y amigos. Por ello, a pesar del protagonismo que adquirieron las críticas de Gasch y Gómez de la Serna, entre otros, las (re)presentaciones de Calder y su circo escultórico en Barcelona y Madrid pueden considerarse como indagaciones en torno a nuevas orientaciones, nuevos lenguajes formales al modo de la vanguardia europea, pero que no llegaron a consolidarse y que como hemos visto sólo fueron accesibles para una minoría.

\section{BIBLIOGRAFÍA}

Brihuega, Jaime (1982): La Vanguardia y la República. Madrid: Ediciones Cátedra.

Calder, Alexander (1966): Calder, an autobiography with pictures. London: Allen Lane The Penguin Press.

Diaz Sanchez, Julián/ Bonet, Juan Manuel/et alt. (2002): Los ismos de Ramón Gómez de la Serna y un apéndice circense. Madrid: Museo Nacional Centro de Arte Reina Sofía, Sociedad Estatal para la Acción Cultural Exterior.

Focius (1933): “A.D.L.A.N. o el Paradís Calderià”. En: La Publicitat, 15 de febrero 1933, Barcelona, s/p.

Gasch, Sebastià (1932a): "El escultor americano Calder". En: $A C$, año II, tercer trimestre, Barcelona, p. 43.

Gasch, Sebastià (1932b): "El circ d'un escultor". En: Mirador, 191, 21 setiembre 1932, Barcelona, p. 5.

Gasch, Sebastià (1933a): “A. Calder”. En: Mirador, 211, 16 febrero 1933, Barcelona, p. 7.

Gasch, Sebastià (1933b): “Alexander Calder, escultor”. En: La Publicitat, 19 de febrero de 1933, Barcelona, p. 9.

Gasch, Sebastià (1933c): “Un ianqui a Barcelona”. En: Mirador, 212, 23 febrero 1933, Barcelona, p. 7.

Gasch, Sebastià (1934): "L'art d'avantguarda a Barcelona". En: D'ací d'allà, número especial de invierno, Barcelona, s/p.

Gasch, Sebastià (1987): Escrits d'art i d'avantguarda (1925-1938). (Edición a cargo de Joan M. Minguet). Barcelona: edicions del Mall.

Giralt-Miracle, Daniel (1992): “ADLAN”. En: Giralt-Miracle, Daniel (1992) (ed.): Las vanguardias en Cataluña 19061939. Barcelona: Fundació Caixa de Catalunya, pp. 354-387.

Gómez de la Serna, Ramón (1933): "Calder y su circo". En: Luz, 1 de febrero de 1933, Madrid, pp. 8-9.

Gray, Cleve (1964): “Calder's Circus”. En: Art in america, 5, pp. 23-33.

Hutton Turner, Elizabeth/Wick, Oliver (eds.) (2004): Calder, Miró. Basel: Foundation Wilson Publishers.

Jané, Jordi/Minguet, Joan M. (1998): Sebastià Gasch, el gust pel circ (antología de textos). Tarragona: El Mèdol.

Johnson Sweeney, James (1964): Alexander Calder, Circus drawings, wire sculpture and toys. Houston: Museum of Fine Arts. Joy Sperling, L. (1988) “Calder in París: The Circus and Surrealisme”. En: Archives of American Art Jounal, 28, pp. 16-29.

Karmel, Pepe (2008): “The Alchemist: Alexander Calder and Surrealism”. En: Simons, Joan/Leal, Brigitte (eds): Alexander Calder. The París years 1926-1933. New Haven: Yale University Press, pp. 212-225.

Martínez-Collaso, Ana (1997): La complejidad de lo moderno. Ramón y el arte nuevo. Cuenca: Ediciones de la Universidad de Castilla-La Mancha.

Meylaters, Reine/Gonne, Maud/Lobbes, Tessa/Sanz Roig, Diana (2017): "Cultural Mediators in Cultural History. What Do We Learn from Studying Mediators' Complex Transfer Activities in Interwar Belgium?”. En: Brems, Elke/ Réthelyi, Orsolya / van Kalmthout, Ton (eds.): Doing Double Dutch: The International Circulation of Literature from the Low Countries. Leuven: Leuven University Press, pp. 67-91.

Minguet, Joan M./Vidal, Jordi (1992): "Vanguardias en Catalunya. Cronología crítica”. En: Giralt-Miracle, Daniel (ed.). Las vanguardias en Cataluña 1906-1939. Barcelona: Fundació Caixa de Catalunya, pp. 466-589.

Minguet, Joan M. (1997): "Un cert malabarisme historiogràfic: ADLAN i les altres arts". En: Bonet, Pilar/Guerrero, Manuel/Minguet, Joan M./Peran, Martí (1997): ADLAN i el circ frediani. Mataró: Patronat Municipal de cultura.

Minguet, Joan M./Montaner, Teresa/Santanach, Joan (2009) (eds.): Epistolari català. Joan Miró 1911-1945. Barcelona: Editorial Barcino, Fundació Joan Miró.

Pérez Ferrero, Miguel (1933): "Un yanqui en la excorte. Hoy, Alejandro Calder ha presentado el circo más pequeño del mundo en la Sociedad de Cursos y Conferencias". En: El Heraldo de Madrid. 1 de febrero de 1933, Madrid, p. 2.

Perl, Jed (2017): Calder. The conquest of time. New York: Alfred A. Knoff.

Rivas Bonillo, Antonio (2012): "Literatura y espectáculo: El circo (1917), de Ramón Gómez de la Serna”. En: Alemany, Rafael/ Chico, Francisco (eds.): Literatura i espectacle = Literatura y espectáculo. Alicante: Biblioteca Virtual Miguel de Cervantes. http://www.cervantesvirtual.com/nd/ark:/59851/bmcht392

Simons, Joan (2008): “Alexander Calder: The París Years”. En: Simons, Joan/Leal, Brigitte (eds.): Alexander Calder. The París years 1926-1933. New Haven: Yale University Press, pp. 25-59.

Zlotescu, Ioana (1998): “Notas a la edición”. En: Zlotescu, Ioana (ed.): R. Gómez de la Serna: Obras completas (vol. III). Barcelona: Galaxia Gutenberg, Círculo de Lectores, pp. 811-819.

Fecha de recepción: 14-X-2018

Fecha de aceptación: 10-IV-2019 